\title{
Lack of collagen XV is protective after ischemic stroke in mice
}

\author{
Hiramani Dhungana ${ }^{1,6}$, Mikko T Huuskonen ${ }^{1,6}$, Taina Pihlajaniemi ${ }^{2}$, Ritva Heljasvaara ${ }^{2,3}$, Denis Vivien ${ }^{4,5}$, Katja M Kanninen $^{1}$, Tarja Malm $^{1}$, \\ Jari Koistinaho ${ }^{1}$ and Sighild Lemarchant ${ }^{* 1}$
}

Collagens are key structural components of basement membranes, providing a scaffold for other components or adhering cells. Collagens and collagen-derived active fragments contribute to biological activities such as cell growth, differentiation and migration. Here, we report that collagen XV knock-out (CoIXV KO) mice are resistant to experimental ischemic stroke. Interestingly, the infarcts of CoIXV KO mice were as small as those of wild-type (WT) mice thrombolysed with recombinant tissue plasminogen activator (rtPA), the actual treatment for ischemic stroke. Importantly, there were no differences in the architecture of cerebrovascular anatomy between WT and CoIXV KO mice. We found a twofold increase of the most potent pro-angiogenic factor, type A vascular growth endothelial factor (VEGF-A) in the ipsilateral cortex of rtPA-treated ischemic WT mice compared with untreated ischemic and sham-operated counterparts. A similar increase of VEGF-A was also found in both rtPA and untreated ischemic ColXV KO mice compared with sham CoIXV KO mice. Finally, we evidenced that the levels of ColXV were increased in the plasma of WT mice treated with rtPA compared with untreated ischemic counterparts. Altogether, this study indicates that the lack CoIXV is protective after stroke and that the degradation of endothelial CoIXV may contribute to the beneficial effect of rtPA after ischemic stroke. The neuroprotection observed in ColXV KO mice may be attributed to the increased VEGF-A production following stroke in the ischemic territory.

Cell Death and Disease (2017) 8, e2541; doi:10.1038/cddis.2016.456; published online 12 January 2017

Stroke is a leading cause of death and long-term disability worldwide. $^{1}$ Ischemic strokes represent $80 \%$ of cerebral strokes and result from the obstruction of a major cerebral artery by a thrombus or an embolus which reduces the blood flow in downstream targeted brain regions, leading to brain damage. The establishment of the primary ischemic lesion is followed by a series of secondary events that worsens tissue damage, including vascular, cellular and molecular events. ${ }^{2}$ Nowadays, the only treatment for ischemic stroke is reperfusion with intravenous administration of recombinant tissue plasminogen activator (rtPA; Alteplase) within a strict time frame up to $4.5 \mathrm{~h}$, leading to an improved functional recovery and reduced neurological deficits. ${ }^{3}$ Additionally to its narrowed therapeutic window, rtPA also increases the risk of intracerebral hemorrhagic transformations and is not efficient at degrading platelet-rich thrombi. Taking together all the limitations for the use of rtPA, only $5 \%$ of ischemic stroke patients are eligible for rtPA-induced thrombolysis. ${ }^{4}$ Therefore, it is essential to better understand stroke pathophysiology in order to find safer and more efficient approaches for therapy.

Collagens are well-known to be critical extracellular components in vascular stability and functions. Some collagens such as CoIXV, ColXVIII and CoIXIX have also been shown to be essential for motor axon guidance and neuromuscular development. ${ }^{5-7}$ The structurally homologous
ColXV and CoIXVIII constitute the so called multiplexin family of non-fibrillar collagens, characterized by multiple triple helix interruptions and similar non-collagenous sequences. ${ }^{8}$ Nevertheless, they differ in their functional properties and expression patterns and for example, contrary to CoIXVIII, CoIXV predominantly carries chondroitin sulfate chains and was therefore classified as a chondroitin sulfate proteoglycan (CSPG) ${ }^{9}$ It is mainly produced by skeletal and cardiac muscle, and endothelial cells, occurring at the basement membranes adjacent to these cells. ColXV knock-out (KO) mice suffer from mild skeletal and cardiac myopathy ${ }^{10,11}$ and defective myelination of peripheral nerves. ${ }^{12}$

In this study, we report for the first time the neuroprotective effect of ColXV deficiency in mice suffering from ischemic stroke. Accordingly, we found an increase of type $A$ vascular endothelial growth factor (VEGF-A) in the ischemic cortex of CoIXV KO mice. Additionally, we showed that rtPA increased the presence of unbound ColXV in the plasma of wild-type (WT) mice after stroke.

\section{Results}

Lack of collagen $\mathrm{XV}$ is protective after thromboembolic stroke. WT and CoIXV KO mice were subjected to thromboembolic stroke provoked by a local injection of thrombin

\footnotetext{
${ }^{1}$ Department of Neurobiology, A. I. Virtanen Institute for Molecular Sciences, Biocenter Kuopio, University of Eastern Finland, Kuopio, Finland; ${ }^{2}$ Oulu Center for Cell-Matrix Research, Biocenter Oulu and Faculty of Biochemistry and Molecular Medicine, University of Oulu, Oulu, Finland; ${ }^{3}$ Centre for Cancer Biomarkers CCBIO, Department of Biomedicine, University of Bergen, Bergen, Norway; ${ }^{4}$ Normandie Univ, UNICAEN, INSERM U919, Serine Proteases and Pathophysiology of the neurovascular Unit, Cyceron, Caen, France and ${ }^{5}$ Department of Clinical Research, Caen University Hospital, Avenue de la côte de Nacre, Caen, France

*Corresponding author: S Lemarchant, Department of Neurobiology, A.I. Virtanen Institute for Molecular Sciences, University of Eastern Finland, PO Box 1627, Kuopio 70211, Finland. Tel: +33 620675 740; Fax: +35 817162 424; E-mail: sighild.lemarchant@uef.fi

${ }^{6}$ These authors contributed equally to this work

Received 25.8.16; revised 02.12.16; accepted 06.12.16; Edited by A Verkhratsky
} 
into the middle cerebral artery as previously described. ${ }^{13,14}$ Twenty minutes after clot formation, mice were treated with intravenous injections of saline or rtPA $\left(10 \mathrm{mg} \mathrm{kg}^{-1}\right)$. Two days after stroke, magnetic resonance imaging (MRI) images revealed that the lesion volume was significantly smaller in ColXV KO mice compared with WT mice (Figures 1a and b: $24.2 \pm 2.5 \mathrm{~mm}^{3}$ for WT mice and $16.3 \pm 1.5 \mathrm{~mm}^{3}$ for ColXV KO mice; $P=0.0179$ ). Interestingly, while early thrombolysis with rtPA was beneficial in WT mice (Figures 1a and b: $13.2 \pm 3.0 \mathrm{~mm}^{3}$ for WT mice injected with rtPA; $P=0.0161$ compared with WT mice injected with saline), no additional benefit of rtPA to ColXV KO mice was observed (Figures 1a and $b: 13.8 \pm 2.3 \mathrm{~mm}^{3}$ for ColXV KO mice injected with rtPA; $P>0.05$ compared with ColXV KO mice injected with saline). Cerebral blood flow (CBF) was monitored by Doppler flowmetry during the surgery in WT and ColXV KO mice treated or not with rtPA. We evidenced a similar reduction of about $90 \%$ of CBF after thrombin injection (clot formation) in WT and ColXV KO mice (Figure 1c: $P>0.05$ between groups after clot formation). While no changes of $\mathrm{CBF}$ were evidenced between clot formation and the end of saline injection in untreated WT and ColXV KO mice (Figure 1c: $P>0.05)$, thrombolysis with rtPA increased CBF in WT (Figure 1c: $10.8 \%$ of initial CBF at clot formation and $41.4 \%$ of initial CBF at the end of rtPA injection in WT mice; $P=0.0352$ ) and ColXV KO mice (Figure 1c: $10.8 \%$ of initial CBF at clot formation and $39.5 \%$ of initial CBF at the end of rtPA injection in ColXV KO mice; $P=0.0474)$.

We then evaluated a set of features that could account for the protection observed in ColXV KO after ischemia. Importantly, we did not observe any difference between the architecture of cerebral vasculatures in the middle cerebral artery area of WT and CoIXV KO healthy mice (Figure 2a). No differences in blood parameters, such as partial pressure $\mathrm{O}_{2}$, partial pressure $\mathrm{CO}_{2}, \mathrm{pH}$ or glucose, were found between WT and ColXV KO mice $20 \mathrm{~min}$ after stroke (Figure 2b). Brain edema has a central role in the pathophysiology of stroke. Therefore, we looked at brain swelling and aquaporin 4, the well-known glial water channel whose expression is increased after stroke and is extensively described for its contribution to brain edema formation and development. ${ }^{15}$ We did not observe any modification of the percentage of swelling between WT and ColXV KO mice treated or not with rtPA 2 days after stroke (Figure 3a: $P=0.2444)$. Similarly, no difference in aquaporin 4 immunoreactivity was found in the peri-ischemic area between WTand ColXV KO mice treated or not with rtPA 3 days after stroke (Figures $3 \mathrm{~b}$ and $\mathrm{c}$ : $P=0.6393)$.

rtPA leads to an increase of collagen XV levels in the plasma of ischemic WT mice. We then measured the protein levels of ColXV in the plasma of sham-operated and ischemic WT mice treated or not with rtPA 3 days after stroke, by ELISA. Stroke did not influence plasma concentrations of ColXV (Figure 4: $P=0.6242$ between sham-operated and untreated ischemic WT mice). However, rtPA tended to increase ColXV protein levels after ischemia in the plasma of WT mice (Figure $4:+1.93 \mu \mathrm{g} \mathrm{ml}^{-1}$ in ischemic WT mice treated with rtPA compared with untreated ischemic WT mice, $P=0.0526)$. a
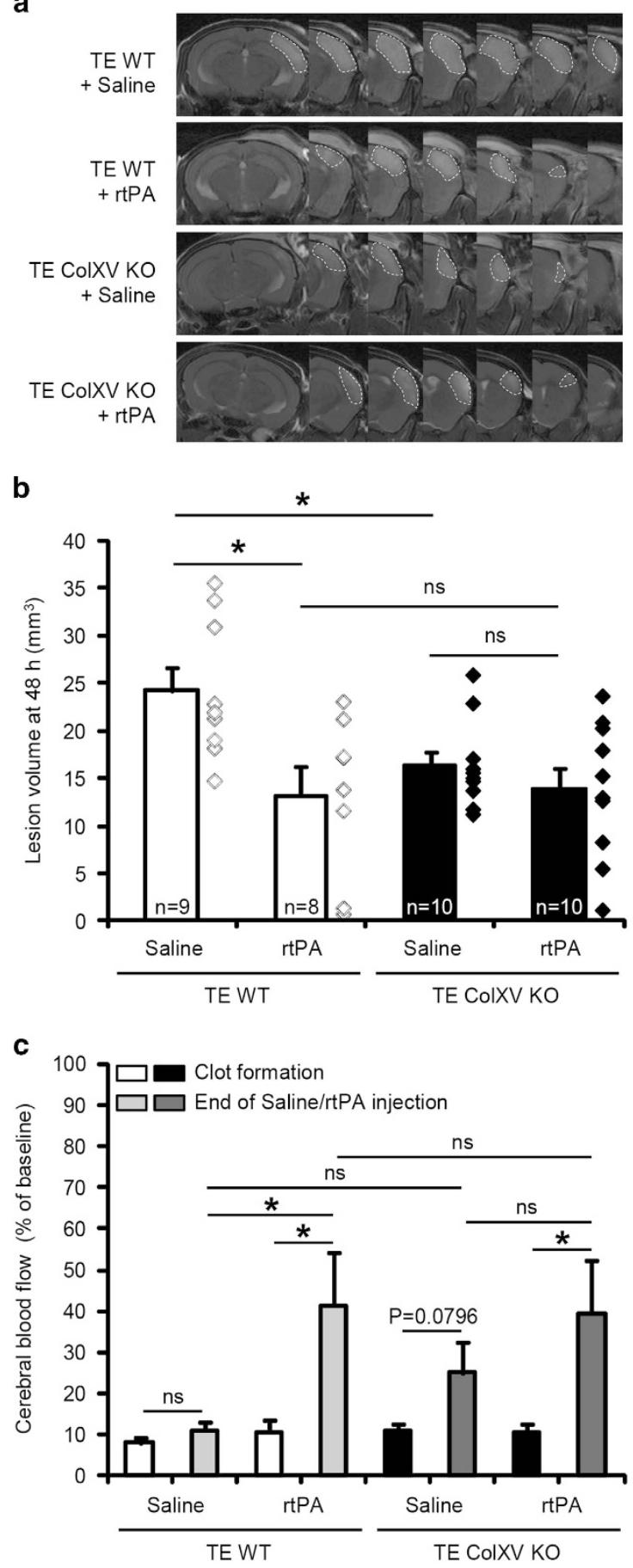

Figure 1 Lack of ColXV is beneficial after thromboembolic stroke. (a) Representative T2-weighted MR images of WT and ColXV KO mice $48 \mathrm{~h}$ after thromboembolic stroke, reperfused or not with rtPA $\left(10 \mathrm{mg} \mathrm{kg}^{-1}\right) 20 \mathrm{~min}$ after thrombin injection. (b) The infarct volume was measured $48 \mathrm{~h}$ after stroke. Values plotted are mean \pm S.E.M. Mann-Whitney U-tests: ${ }^{*} P<0.05$ relative to WT+Saline; $N=8-10$ per group. (c) Monitoring of cerebral blood flow registered during surgical approach by Doppler flowmetry. No differences in Doppler reduction after thrombin injection (clot formation) or rtPA-induced recanalization (at the end of Saline/rtPA injection) were noticed between WT and ColXV KO mice. Values plotted are mean \pm S.E.M. Mann-Whitney U-tests: ${ }^{*} P<0.05 ; N=8-10$ per group 
To determine why ColXV KO mice are more resistant to ischemic stroke than WT mice, we then investigated whether the lack of ColXV could influence mechanisms mediating neuroinflammation or neuroprotection.

rtPA fails to reduce stroke-induced increases of interleukin- 6 and chemokine ligand 2 protein levels in ischemic collagen XV KO mice. First, we did not observe any changes in astrogliosis or microgliosis between WT and ColXV KO mice treated or not with rtPA 3 days after stroke, measured respectively as GFAP (Figures $5 a$ and b: $P=0.8330$ ) or lba1 (Figures $5 \mathrm{c}$ and $\mathrm{d}: P=0.6932$ ) reactivity in the peri-ischemic area.

In a parallel cohort of animals, we investigated the expression of cytokines present in protein extracts from the contralateral and ipsilateral cortices of WT and CoIXV KO mice treated or not with rtPA 3 days after stroke. Cytometric bead assay revealed a significant increase of interleukin-6 (IL-6) or chemokine ligand 2 (CCL2) protein levels in the ipsilateral cortex of mice suffering from stroke compared with corresponding contralateral cortex or to corresponding sham-operated mice (Figures $6 a$ and b). We observed that thrombolysis with rtPA led to a twofold decrease of stroke-induced IL-6 (Figure 6a: $-58.7 \mathrm{pg} \mathrm{ml}^{-1}$ in ischemic WT mice treated with rtPA compared with untreated ischemic WT mice, $P=0.0143$ ) and CCL2 (Figure 6b: $-288.7 \mathrm{pg} \mathrm{ml}^{-1}$ in ischemic WT mice treated with rtPA compared with untreated ischemic WT mice, $P=0.0500$ ) increases in the ipsilateral cortex of WT mice. Interestingly, rtPA failed to reduce stroke-induced IL-6 (Figure 6a: $P=0.6242$ ) and CCL2 (Figure 6b: $P>0.9999)$ increases in the ipsilateral cortex of ColXV KO mice. There were no differences of IL-6 (Figure 6c: $P=0.2506$ ) or CCL2 (Figure 6d: $P=0.6015$ ) protein levels in the ipsilateral cortex of WT and ColXV KO mice. No modifications of tumor necrosis factor a (TNF-a) and interferon $\gamma(\mathrm{IFN}-\gamma)$ protein levels were found between

a

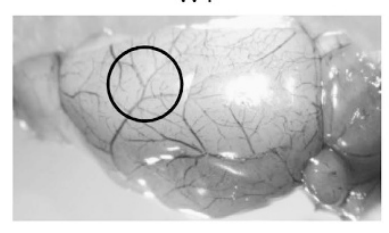

\begin{tabular}{lll} 
b Blood parameters & WT & ColXV KO \\
\hline Partial pressure $\mathrm{O}_{2}(\mathrm{mmHg})$ & $49.75 \pm 2.06$ & $50.67 \pm 5.03$ \\
Partial pressure $\mathrm{CO}_{2}(\mathrm{mmHg})$ & $58.63 \pm 5.51$ & $62.33 \pm 5.75$ \\
$\mathrm{pH}$ & $6.99 \pm 0.10$ & $6.99 \pm 0.06$ \\
Glucose $(\mathrm{mmol} / \mathrm{L})$ & $7.50 \pm 1.50$ & $5.73 \pm 0.80$
\end{tabular}

Figure 2 No differences of blood parameters and cerebral vasculatures between WT and ColXV KO mice. (a) Measurements of blood parameters in WT $(N=4)$ and ColXV KO $(N=3)$ mice 20 min after thromboembolic stroke. No significant difference in any of these parameters was noted between WT and CoIXV KO mice. (b) Cerebral vasculature of WT and CoIXV KO mice. Black circles indicate the middle cerebral artery bifurcation the groups (Figures $6 \mathrm{c}$ and $\mathrm{d}$ : $P=0.3841$ for TNF- $a$, $P=0.3570$ for IFN- $\gamma$ ). IL-10 and IL-12p70 were not detected in the samples.

a
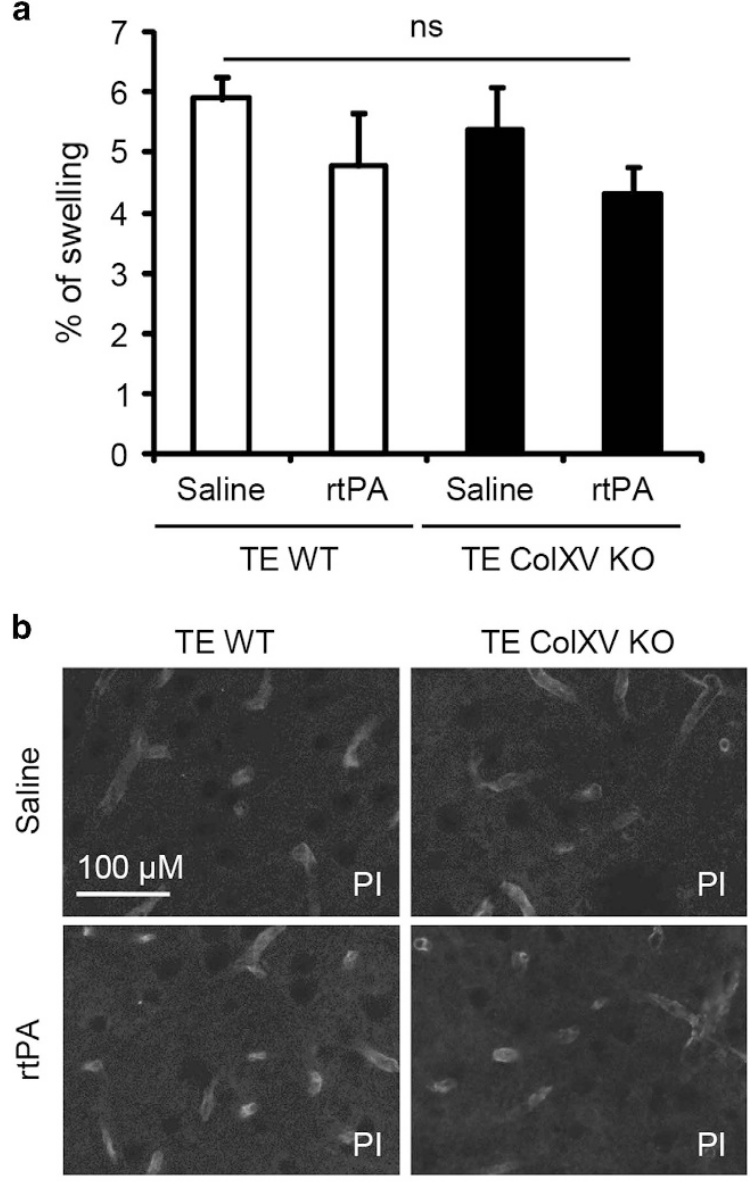

C
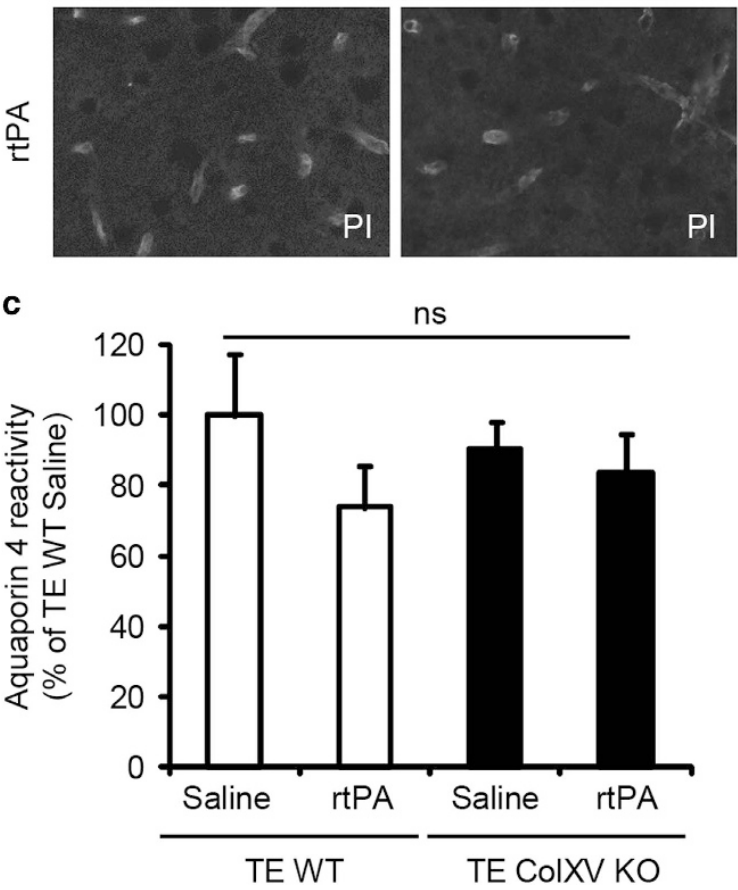

Figure 3 Lack of ColXV does not influence brain edema. (a) Brain edema was measured as a \% of swelling by using the formula ( $(\mathrm{V}$ ipsilateral hemisphere $-\mathrm{V}$ contralateral hemisphere) $/ V$ contralateral hemisphere) ${ }^{*} 100$. Values plotted are mean \pm S.E.M. Kruskall-Wallis test: $P>0.05$. (b-c) Photomicrographs of aquaporin 4 (b) and corresponding immunoreactivity quantifications (c) in the peri-ischemic area of injured WT and ColXV KO mice thrombolysed or not with rtPA 20 min after thromboembolic (TE) stroke. Values plotted are mean \pm S.E.M. Kruskall-Wallis test: $P>0.05 ; N=4-5$ per group. $\mathrm{Pl}$, peri-ischemic area 
VEGF-A expression is increased in the ischemic cortex of collagen XV KO mice after thromboembolic stroke. We then studied the expression of VEGF-A, a neuroprotective molecule in the $\mathrm{CNS}^{16}$ including after ischemic stroke. ${ }^{17}$

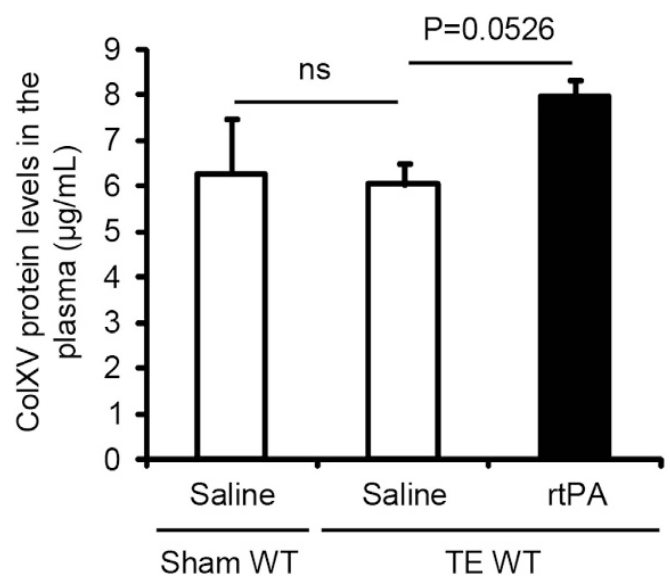

Figure 4 rtPA leads to an increase of ColXV levels in the plasma of WT mice. ELISA for mouse ColXV in the plasma of sham-operated and ischemic WT mice thrombolysed or not with rtPA 20 min after thromboembolic (TE) stroke, at 3 days post injury. Values plotted are mean \pm S.E.M. Mann-Whitney U-tests: $P=0.0526 ; N=3-5$ per group
We observed a twofold increase of VEGF-A in the ipsilateral cortex of ischemic WT mice treated with rtPA compared with untreated ischemic WT mice or sham-operated WT counterparts (Figures $7 a$ and $b:+110.7 \%$ in ischemic WT mice treated with rtPA compared with untreated ischemic WT mice, $P=0.0209$ ). Interestingly, we observed a similar increase of VEGF-A in ischemic ColXV KO mice treated or not with rtPA compared with sham-operated ColXV KO counterparts (Figures 7c and $\mathrm{d}$ : $+131.9 \%$ in ischemic ColXV KO mice compared with sham-operated ColXV KO counterparts, $P=0.0209 ; P=0.3865$ between ischemic ColXV KO mice treated or not with rtPA).

\section{Discussion}

Collagens, laminins, nidogens and perlecan are major structural proteins of basement membranes, self-assembled with other extracellular matrix (ECM) components, which altogether represent a complex network providing a crucial molecular and physical scaffold for cells. ${ }^{18}$ Here, we investigated for the first time the role of ColXV in the CNS after acute ischemic stroke in mice. Interestingly, we demonstrated that ColXV-deficient mice are more resistant to thromboembolic stroke than WT siblings. Indeed, we observed a 33\% decrease

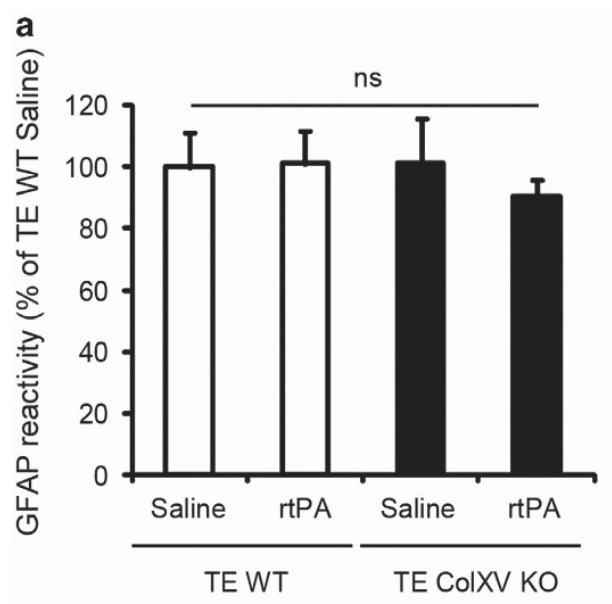

b
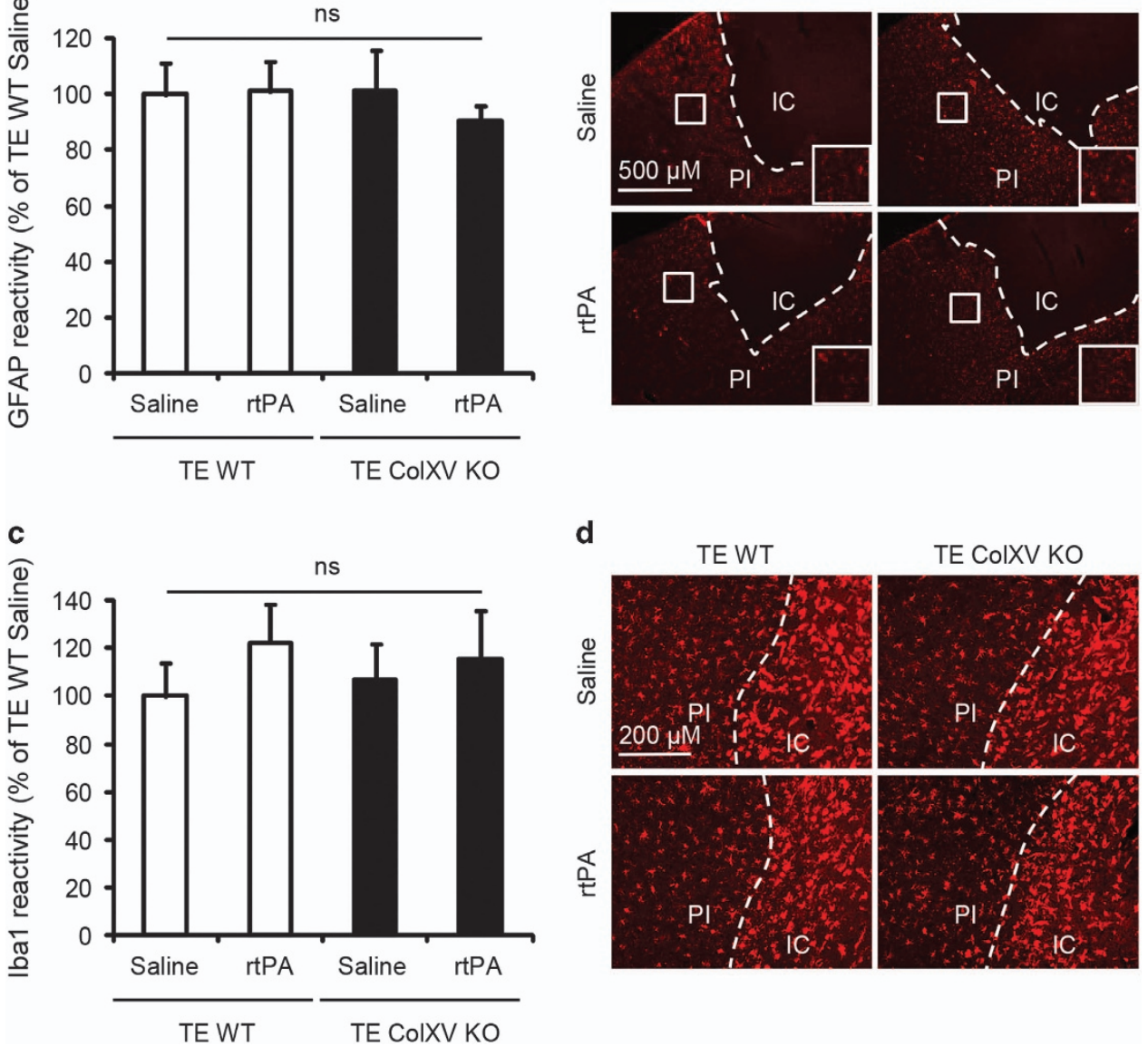

d

d TE WT
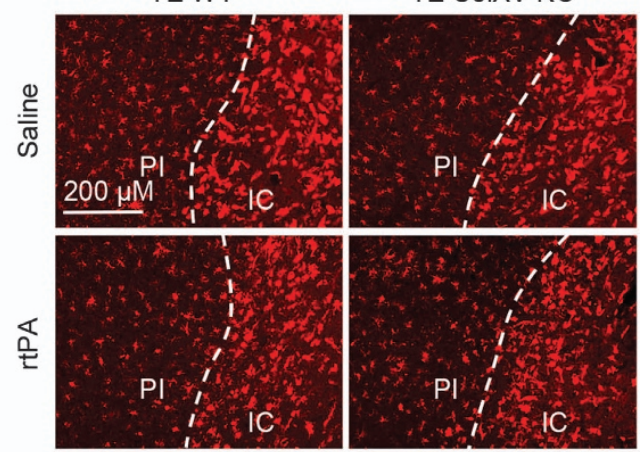

Figure 5 Lack of ColXV does not influence gliosis. Photomicrographs of GFAP (a), Iba1 (c) and corresponding immunoreactivity quantifications (b,d) in the peri-ischemic area of injured WTand ColXV KO mice thrombolysed or not with rtPA 20 min after thromboembolic (TE) stroke. Values plotted are mean \pm S.E.M. Kruskall-Wallis test: $P>0.05$; $N=4-5$ per group. IC, ischemic core; $\mathrm{PI}$, peri-ischemic area 

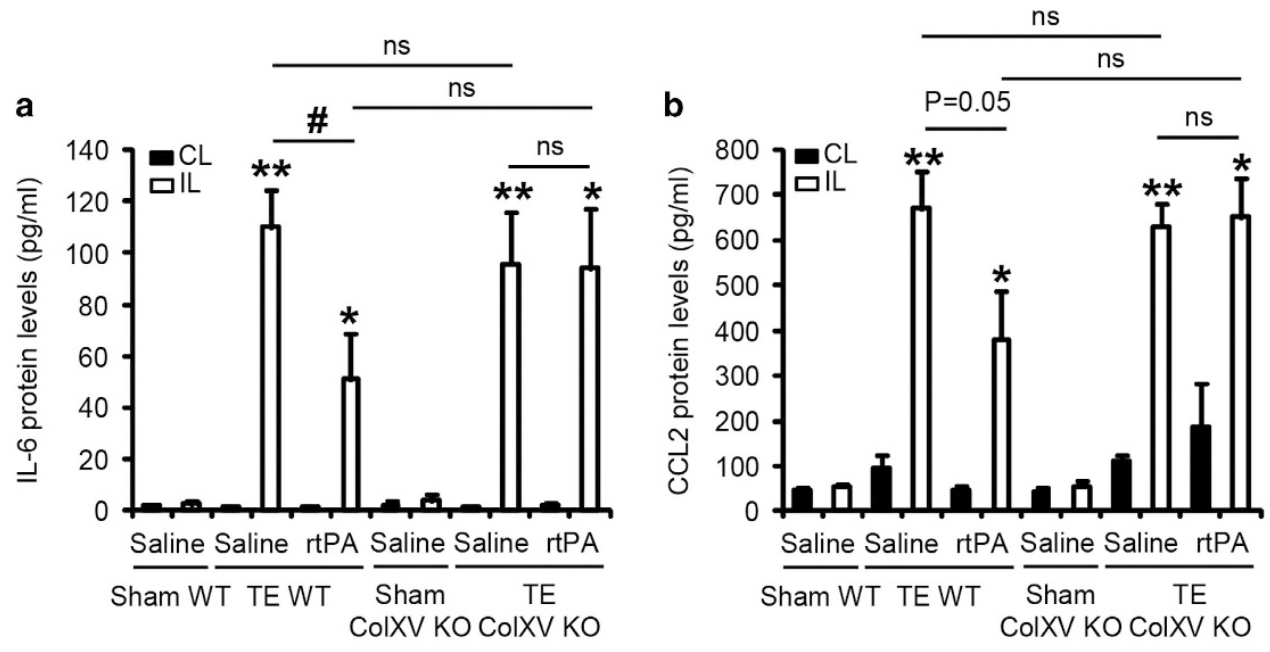

c

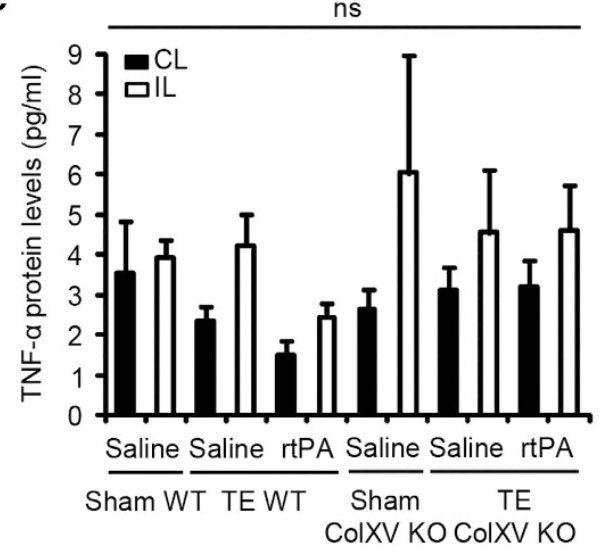

d

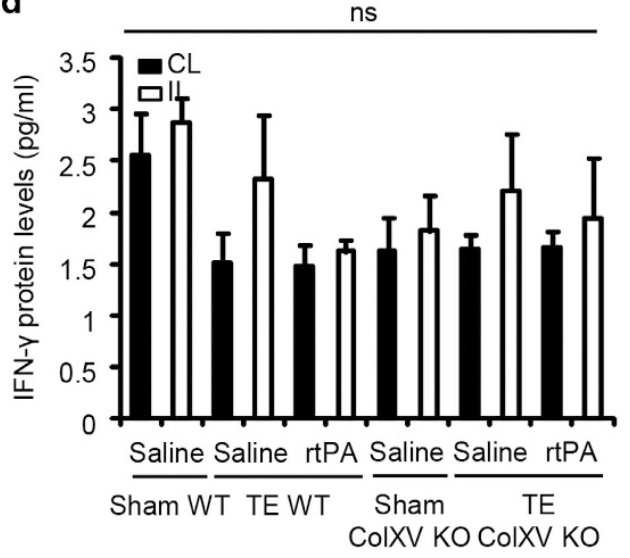

Figure 6 rtPA fails to reduce stroke-induced increases of IL-6 and CCL2 protein levels in the ischemic cortex of ColXV KO mice. IL-6 (a), CCL2 (b), TNF- $\alpha$ (c) and IFN- $\gamma$ (d) protein levels at 3 days post injury in the contralateral (CL, black bars) and ipsilateral (IL, white bars) cortices of sham-operated and injured WTand CoIXV KO mice thrombolysed or not with rtPA 20 min after TE stroke. Values plotted are mean \pm S.E.M. Kruskal-Wallis: $P>0.05$ for TNF- $\alpha$ and IFN- $\gamma, P<0.0001$ for IL-6 and CCL2. Mann-Whitney U-tests: ${ }^{\star} P<0.05,{ }^{* \star} P<0.01$ compared to the contralateral hemisphere, ${ }^{\#} P<0.05$ compared to the ipsilateral cortex of saline TE WT; $N=4-5$ per group
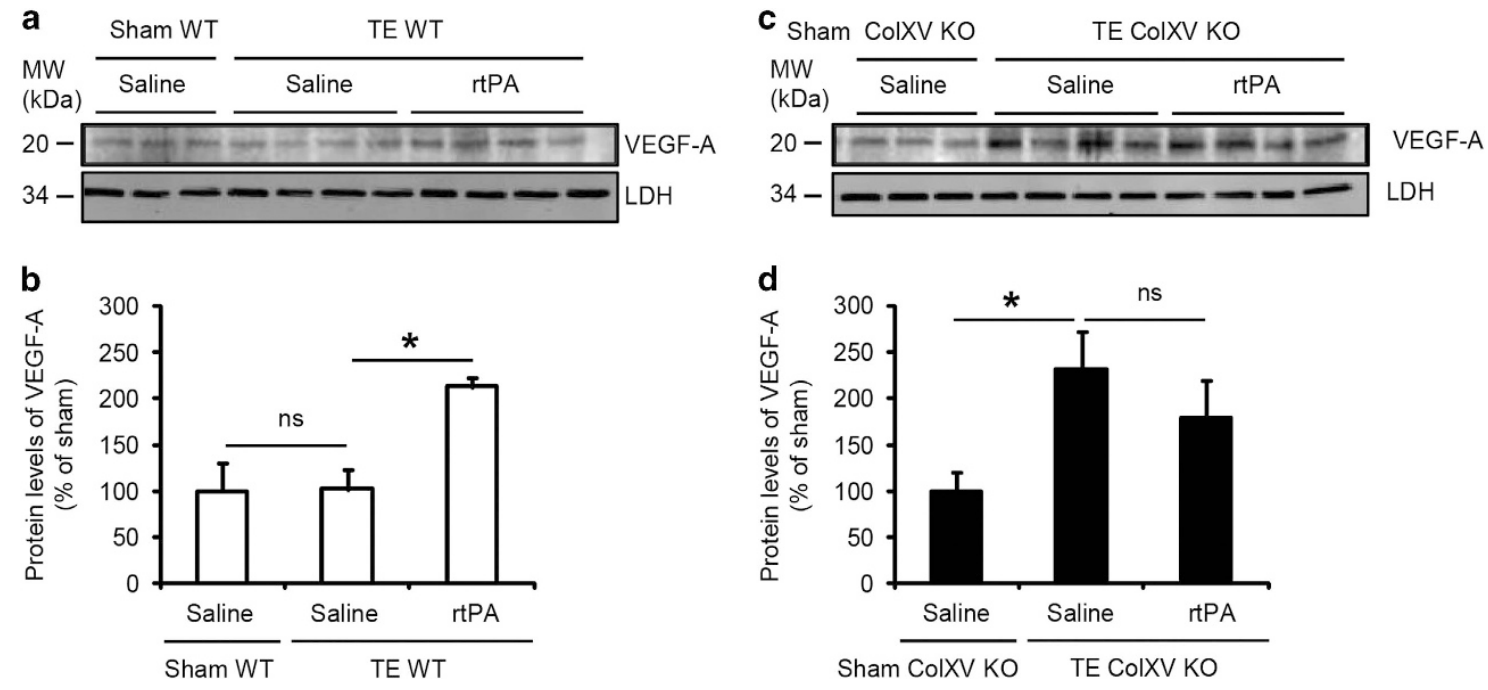

Figure 7 Lack of CoIXV increases VEGF-A expression in the ischemic cortex. Immunoblots for VEGF-A (a and $\mathbf{c}$ ) and corresponding quantifications (b and $\mathbf{d})$ in the ipsilateral cortex of sham-operated and injured WT ( $\mathbf{a}$ and $\mathbf{b}$ ) and ColXV KO (c and $\mathbf{d}$ ) mice thrombolysed or not with rtPA 20 min after thromboembolic (TE) stroke, at 3 days post injury. Values plotted are mean \pm S.E.M. Mann-Whitney U-tests: * $P<0.05 ; N=4$ 
in the cortical lesion volume of ColXV-deficient mice compared with WT mice, associated with a twofold increase of VEGF-A in the ischemic cortex. Interestingly, we also showed that rtPA tends to increase the plasma levels of ColXV in ischemic WT mice. Altogether, this study suggests the involvement of ColXV in deleterious pathways occurring during stroke that contribute to worsen brain damage.

ColXV is present in numerous tissues, synthesized by endothelial cells, myoblasts, fibroblasts and epithelial cells, and located at vascular, neuronal, mesenchymal or epithelial basement membrane zones. ${ }^{19}$ Due to its privileged location at the basement membrane zone, ColXV is crucial for the structural integrity of skeletal muscle cells and capillaries. ${ }^{10,11}$ Indeed, although ColXV KO mice are viable, they develop progressive mild muscle degeneration and abnormal microvessels in heart and skeletal muscles specifically. ${ }^{10,11}$ Accordingly to the same report, ${ }^{11}$ we did not find any difference in the cerebral vasculature between WT and CoIXV $\mathrm{KO}$ mice. Surprisingly, one could have expected that ischemic stroke would have worsened brain damage in ColXV KO mice, by weakening the vasculature. On the contrary, ColXV KO mice presented a considerably smaller lesion volume compared with WT mice, with an efficacy similar to the actual treatment for acute ischemic stroke, rtPA. ${ }^{3}$ Unexpectedly, we did not observe any synergic or additional benefit of rtPA in ColXV KO mice. Basement membrane components have the ability to bind other ECM components such as cell surface receptors, cytokines or growth factors. ${ }^{18}$ Therefore, we hypothesized that the basement membranes of ColXV KO mice may have lost their ability to bind and sequester some of these ECM components due to their new structural composition and/or to the absence of ECM component interactions with the chondroitin sulfate chains of ColXV. It could explain VEGF-A over-expression in ischemic CoIXV KO mice. Similarly, it would explain the finding that rtPA failed to decrease stroke-induced increases of IL-6 and CCL2 protein levels in ischemic ColXV KO mice. This result suggests that the reduced production of these cytokines by rtPA may be triggered by ColXV. The expression of CCL2 and IL- 6 are increased centrally and peripherally after stroke in animal models and in human patients. ${ }^{20-23}$ Therapies aiming the blockade of CCL2 via a gene transfer or a neutralizing antibody were protective in rodent models of transient middle cerebral artery occlusion (tMCAO). ${ }^{20,24}$ Similarly, CCL2 KO mice had a smaller lesion volume compared with WT mice after experimental stroke associated with a reduced bloodbrain barrier leakage, a decreased infiltration of leukocytes and a decreased production of pro-inflammatory cytokines. ${ }^{25-27}$ On the contrary, transgenic mice overexpressing CCL2 presented a large ischemic lesion compared with WT mice after permanent MCAO (pMCAO). ${ }^{28}$ Contrary to CCL2, no consensus about the effect of IL- 6 after stroke was established to explain the discrepancies observed in animal models and in human patients. Although injection of IL- 6 was protective after pMCAO in rats, ${ }^{29}$ the lesion volumes of IL- $6 \mathrm{KO}$ and WT mice were similar after $\mathrm{MCAO}$ in mice. ${ }^{30}$ Conversely, increased levels of IL-6 in the serum of human patients correlated with more severe neurological outcomes and a poor diagnosis. $^{22-23}$
The collagen matrix is continuously remodeled by proteasedependent collagen degradation and production of new collagens. In this regard, our results suggest the possible cleavage of endothelial ColXV by rtPA, possibly participating to the rtPA beneficial effects in ischemic WT mice. This hypothesis is comforted by previous reports showing the ability of rtPA to cleave/degrade CSPGs. ${ }^{31,32}$ Proteolytic cleavages of ColIV, ColXV and ColXVIII produce fragments with anti-angiogenic activities: arrestin, canstatin or tumstatin for ColIV, restin for ColXV and endostatin for ColXVIII. ${ }^{33,34}$ Increasing post-stroke repair constitutes one direction of interest to establish new effective therapies. In this regard, VEGF-A is of particular interest for its involvement in mechanisms mediating neuroprotection, angiogenesis, neurogenesis, neuronal migration and survival and axon guidance. $^{16,17}$ Nevertheless, previous studies caution the use of VEGF for stroke therapy at appropriate dose and time post-stroke. ${ }^{35}$ Here, we have focused on short-term time points: MRI was conducted at 2 days post injury, and the mice were sacrificed one day later. Considering the anti-angiogenic effect of restin and the pro-angiogenic effect of VEGF-A, further investigations are now needed to study a long-term time point, to establish whether the increased VEGF-A production observed early after stroke is not transient but promotes long-lasting angiogenesis in ColXV KO mice after ischemic stroke. In line with our study, compelling evidence shows that CSPGs and heparan sulfate proteoglycans (HSPGs) have critical roles in mechanisms mediating neuroprotection and angiogenesis after stroke. Deglycosylation of CSPGs by the bacterial enzyme chondroitinase $A B C$ reduced glial scar formation, promoted axonal regeneration and collateral sprouting, and improved functional outcome after experimental stroke in rodents. ${ }^{36,37}$ Similarly, the HSPG glycipan also reduced glial scar and improved functional outcome of rats after pMCAO. ${ }^{36}$ Additionally, chondroitinase $A B C$ and glycipan increased the production of neurotrophic factors in primary cultures of cortical neurons (brain-derived neurotrophic factor for chondroitinase $A B C$ and fibroblastgrowth factor 2 for glycipan). ${ }^{36}$ The C-terminal protein fragment domain $\mathrm{V}$ of the HSPG perlecan reduced the infarct size and enhanced angiogenesis in a VEGF-dependent manner in the peri-ischemic area thereby promoting functional recovery in several rodent models of ischemic stroke. ${ }^{37,38}$

This study indicates that the lack of ColXV is protective after ischemic stroke. The neuroprotection observed in ColXVdeficient mice may be attributed to the acutely increased production of a neuroprotective molecule, VEGF-A, ${ }^{16,17,39}$ following stroke in the ischemic territory. Further studies are now warranted to decipher the mechanism(s) underlying VEGF-A production in ColXV KO mice during stroke, to determine the cellular origin of VEGF-A (endothelial cells? neurons? glia?), and finally, to investigate whether the increased production of VEGF-A in ColXV-deficient mice may increase angiogenesis during the chronic phase of ischemic stroke. This study also highlights that ColXV may represent a substrate for rtPA, the actual treatment of ischemic stroke. 


\section{Materials and Methods}

Ethics. Animal experiments were conducted according to the national regulation of the usage and welfare of laboratory animals, approved by the National Animal Experiment Board of Finland and followed the Council of Europe legislation and regulation for animal protection.

Animals. Adult WT and transgenic female mice lacking in the $\alpha 1$ chain of collagen XV (ColXV KO) by site-specific Cre-loxP-mediated deletion in embryonic stem cells from C57BL6J background were used in this study. ${ }^{11}$ Transgenic genotypes were identified by PCR amplification of ear DNA a few days after birth and after death to confirm the results of the first genotyping. The mice were housed under controlled temperature, humidity and light conditions (12 h light and dark cycles) with free access to food and water. Animals were housed in groups of up to 5 in cages.

Thromboembolic stroke model. Nine month-old WT and ColXV KO female mice were anesthetized by $5 \%$ isoflurane in $30 \% \mathrm{O}_{2} / 70 \% \mathrm{~N}_{2} \mathrm{O}$ and the surgical anesthesia was maintained by $2 \%$ isoflurane. Temperature was maintained at $37 \pm 1^{\circ} \mathrm{C}$ by thermostatically controlled heating blanket (Harvard apparatus; PanLab, Cornella, Spain). In a parallel group, a femoral artery was catheterized for monitoring of blood gases $\left(p H, p \mathrm{O}_{2}, p \mathrm{CO}_{2}\right) 20$ min after ischemia. Mice were placed in a stereotaxic frame, the skin between the right eye and the right ear was incised, and the temporal muscle was retracted. A small craniotomy was performed, the dura was excised, and the middle cerebral artery (MCA) was exposed. One $\mu \mathrm{L}$ containing $1 \mathrm{IU}$ purified murine $\alpha$-thrombin (Enzyme Research Laboratories, South Bend, IN, USA) was injected into the MCA using a micropipette to induce the formation of a stable clot in situ. ${ }^{13,14}$ Cerebral blood velocity in the MCA territory was followed during the surgery by laser Doppler (Moor Instruments, Axminster, UK) to confirm the drop of the blood flow after the clot formation. Thrombolysis was induced after $30 \mathrm{~min}$ of occlusion by injecting $200 \mu \mathrm{l}$ of rtPA (10 mg/Kg; Actilyse, Boerhinger Ingelheim, Germany) intravenously into the tail vein (10\% bolus, $90 \%$ perfusion for $40 \mathrm{~min}$ ). Control mice received $200 \mu \mathrm{l}$ of saline under the same conditions. Mice were randomized into six groups using GraphPad Quickcalcs (GraphPad Software, La Jolla, CA, USA): Sham-operated WT and ColXV KO mice, untreated thromboembolic (TE) stroked WT and ColXV mice, and rtPA-treated TE stroked WT and ColXV mice.

For the first study, mice were sacrificed 3 days after stroke by terminal perfusion with heparinized saline, brains were dissected and the contralateral and ipsilateral cortices were collected and stored at $-70^{\circ} \mathrm{C}$ for protein purposes $(N=4-5$ in each group). For the second study, mice were sacrificed 3 days after stroke by terminal perfusion with heparinized saline followed by a perfusion with paraformaldehyde (PFA; as described in the immunohistochemistry section) for staining purposes ( $N=4-5$ in each group).

Magnetic resonance imaging. MRI was performed in vivo at 2 days post injury in anesthetized mice to determine the lesion volume using a horizontal 9.4 T Oxford NMR 400 magnet (Oxford instrument PLC, Abington, UK) as previously described. ${ }^{40}$ Multi-slice T2-weighted images were acquired: echo time/repetition time of $40 \mathrm{~ms} / 3000 \mathrm{~ms}$, matrix size $128 \times 256$, field of view $19.2 \times 19.2 \mathrm{~mm}^{2}$, slice thickness $0.8 \mathrm{~mm}$ and number of slices 12 . Images were then analyzed using Aedes software (Kuopio, Finland) under MatLab program (Math-works, Natick, USA).

Protein extraction. Contralateral and ipsilateral cortices were dissociated in ice-cold TNT buffer (50 mM Tris-HCl pH 7.4; $150 \mathrm{mM} \mathrm{NaCl}$; 0.5\% Triton X-100) containing EDTA/EGTA (ethylene diamine/glycol tetraacetic acid, $1 \mathrm{mM}$ ), protease (Sigma-Aldrich, St. Louis, MO, USA) and phosphatase (Roche Diagnostics, Mannheim, Germany) inhibitors. Debris were removed by centrifugation $(12000 \times \mathrm{g}$ at $4{ }^{\circ} \mathrm{C}, 15 \mathrm{~min}$ ). Supernatants were stored at $-70^{\circ} \mathrm{C}$ until further processing. Protein quantification was performed according to the BCA protein method (Pierce, Rockford, USA)

Western blot. Proteins $(5 \mu \mathrm{g})$ were resolved on $14 \%$ polyacrylamide gel under denaturing conditions and transferred onto a polyvinylidene difluoride membrane. Membranes were blocked with phosphate-buffered saline (PBS) tween $(0.2 \%$ Tween-20; Sigma-Aldrich) and $5 \%$ of milk. Blots were incubated overnight at $4{ }^{\circ} \mathrm{C}$ with the mouse anti-VEGF-A (1/500; Santa Cruz Biotechnology, Dallas, TX, USA) primary antibody diluted in PBS-tween containing $5 \%$ of milk. After a $2-\mathrm{h}$ incubation at room temperature (RT) with the peroxidase-conjugated anti-mouse secondary antibody (1/5000; GE Healthcare Life Sciences, Uppsala, Sweden), proteins were revealed with an enhanced chemiluminescence ECL-Plus kit immunoblotting detection system (GE Healthcare Life Sciences) and visualized using Storm Fluorlmager system. Rabbit anti-LDH (1/500; Santa Cruz Biotechnology) was used as a loading control $\left.\right|^{41,42}$ and visualized by Alexa fluor 647conjugated anti-rabbit secondary antibody (1/1000; Jackson ImmunoResearch Laboratories, West Grove, PA, USA).

Immunohistochemistry. Anesthetized mice were perfused with cold heparinized saline, followed by a perfusion with 4\% PFA in $0.1 \mathrm{M}$ phosphate buffer (PB) pH 7.4. Brains were collected and rinsed in a PB containing $20 \%$ sucrose for cryoprotection for $24 \mathrm{~h}$ and then embedded and frozen in OCT (Optimal Cutting Temperature; Sakura Finetek, Tokyo, Japan). Six 20- $\mu$ m-coronal sections $200 \mu \mathrm{m}$ apart of each brain were cut on a cryostat (Leica Microsystems, Wetzlar, Germany), collected on poly-lysine glasses (Thermo Scientific, Leicestershire, UK), and stored at $-70^{\circ} \mathrm{C}$ until analysis. After washing with PB, PBS and PBS-tween $(0.05 \%$ Tween-20), sections were treated when required with PBS-Triton X-100 $(0.4 \%$, Sigma-Aldrich) and unspecific bindings were blocked by 1 -h incubation in $10 \%$ normal goat serum (NGS, Merck Millipore, Billerica, MA, USA). Incubation with primary antibodies was conducted overnight at RT with dilutions as follows: rabbit anti-GFAP (glial fibrillary acidic protein, 1/200; Dako), rabbit anti-lba1 (ionized calcium-binding adapter molecule-1, 1/250; Wako Pure Chemical Industries, Tokyo, Japan) or rabbit anti-aquaporin 4 (1/300; Merck Millipore). After washing with PBStween, sections were incubated with corresponding fluorescent Alexa fluor -488 or -568-conjugated secondary antibodies (1/200; Life Technologies) for $2 \mathrm{~h}$ at RT, then washed again and finally mounted in Vectashield with DAPI (Vector Laboratories, Burlingame, CA, USA). Negative controls for unspecific binding of the secondary antibodies were conducted in parallel sections following the same procedures described above, except the incubation in primary antibodies.

For the analyses, the peri-ischemic area was imaged using 10x magnification on an AX70 microscope (Olympus corporation, Tokyo, Japan) coupled to a digital camera (Color View 12, soft Imaging System, Muenster, Germany) using Soft Imaging software. Immunoreactivity were quantified using Image-Pro Plus software (Media Cybernetics, Rockville, MO, USA) at a pre-defined range, measured as the relative immunoreactive area for GFAP, Iba1or aquaporin 4 . Analysis were performed blinded to the study groups ( $N=4-5$ in each group).

CoIXV ELISA. Terminal blood samples were collected from the heart into tubes containing 10\% vol/vol $3.8 \%$ trisodium citrate (pH 5.2 adjusted with citric acid). Plasma samples were collected after two consecutive centrifugations, one for $15 \mathrm{~min}$ at $2000 \times \mathrm{g}$ and one or three min at $12000 \times g$. ELISA for mouse ColXV was performed according to the manufacturer's instructions (LSBio, Seattle, WA, USA) using plasma samples diluted 1:2.

Cytokine protein expression. IL-6, CCL2, TNF- $\alpha$, IFN- $\gamma$, IL-10 and IL-12p70 proteins contained in protein lysates from the contralateral and ipsilateral cortices of sham-operated and ischemic WT and ColXV KO mice treated or not with tPA were measured by using the cytometric bead assay Th1/Th2/Th17 kit (BD Biosciences, Franklin Lakes, NJ, USA) according to the manufacturer's instructions. Data were acquired using FACSCalibur (BD Biosciences, San Jose, CA, USA) and analyzed by FCAP Array software (Soft Flow, St. Louis Park, MN, USA).

Middle cerebral artery territory. To evaluate the cerebrovascular anatomy of WT and CoIXV KO mice, anesthetized mice were perfused with saline as described in the IHC section, then with a diluted India blue ink dye solution and finally with PFA. The brains were dissected out and the images were taken using stereomicroscope (Ziess, Oberkochen, Germany) to determine the difference in vasculature around the MCA territory.

Statistical analyses. The data are expressed as mean \pm S.E.M. An alpha level of $P<0.05$ was used for determination of significance in all statistical tests. Statistical analyses were performed with the Statview software package (v5.0). Kruskal-Wallis test was used for intergroup multiple comparisons. In significant cases, Mann-Whitney U-test was applied as post hoc test.

\section{Conflict of Interest}

The authors declare no conflict of interest. 
Acknowledgements. We thank Mrs Mirka Tikkanen and Mrs Velta KeksaGoldsteine for their technical assistance, Drs Yuriy Pomeshchick and Paula Korhonen for their help with transcardiac perfusions, and Dr. Jérôme Parcq and Mathilde Pruvost for unpublished materials. We also thank the Biocenter Oulu Transgenic Facility and the University of Oulu animal facility for expert help. This work was supported by the Council for Health Sciences and the Centre of Excellence Program 2012-2017 of the Academy of Finland (Grant 251314), the INSERM, the ERANETNeuron research program 'ProteA: Proteases before, during and after stroke', 2012-2015, and the Sigrid Jusélius Foundation.

\section{Author contributions}

JK and SL designed research, analyzed and/or interpreted the data. HD, MH, KK, TM and SL performed experiments. TP, RH and DV provided mice and materials. SL wrote the article, and JK, TP, DV and KK gave critical comments on the draft of the manuscript. All authors read and approved the final version of the manuscript.

1. Donnan GA, Davis SM. Breaking the $3 \mathrm{~h}$ barrier for treatment of acute ischaemic stroke. Lancet Neurol 2008; 7: 981-982.

2. George PM, Steinberg GK. Novel stroke therapeutics: unraveling stroke pathophysiology and its impact on clinical treatments. Neuron 2015; 87: 297-309.

3. The National Institute of Neurological Disorders and Stroke rt-PA Stroke Study Group. Tissue plasminogen activator for acute ischemic stroke. N Engl J Med 1995; 333 1581-1587.

4. Hacke W, Lichy C. Thrombolysis for acute stroke under antiplatelet therapy: safe enough to be beneficial? Nat Clin Pract Neurol 2008; 4: 474-475.

5. Guillon E, Bretaud S, Ruggiero F. Slow muscle precursors lay down a collagen XV matrix fingerprint to guide motor axon navigation. J Neurosci 2016; 36: 2663-2676.

6. Hilario JD, Wang C, Beattie CE. Collagen XIXa1 is crucial for motor axon navigation at intermediate targets. Development 2010; 137: 4261-4269.

7. Schneider VA, Granato M. The myotomal diwanka (lh3) glycosyltransferase and type XVIII collagen are critical for motor growth cone migration. Neuron 2006; 50: 683-695.

8. Ricard-Blum S, Ruggiero F. The collagen superfamily: from the extracellular matrix to the cell membrane. Pathol Biol 2005; 53: 430-442.

9. Li D, Clark CC, Myers JC. Basement membrane zone type XV collagen is a disulfide-bonded chondroitin sulfate proteoglycan in human tissues and cultured cells. J Biol Chem 2000; 275 : 22339-22347.

10. Rasi K, Piuhola J, Czabanka M, Sormunen R, Ilves $\mathrm{M}$, Leskinen $\mathrm{H}$ et al. Collagen XV is necessary for modeling of the extracellular matrix and its deficiency predisposes to cardiomyopathy. Circ Res 2010; 107: 1241-1252.

11. Eklund L, Piuhola J, Komulainen J, Sormunen R, Ongvarrasopone C, Fássler R et al. Lack of type XV collagen causes a skeletal myopathy and cardiovascular defects in mice. Proc Natl Acad Sci USA 2001; 98: 1194-1199.

12. Rasi K, Hurskainen M, Kallio M, Stavén S, Sormunen R, Heape AM et al. Lack of collagen XV impairs peripheral nerve maturation and, when combined with laminin-411 deficiency, leads to basement membrane abnormalities and sensorimotor dysfunction. J Neurosci 2010; 30 : 14490-14501.

13. Orset C, Macrez R, Young AR, Panthou D, Angles-Cano E, Maubert E et al. Mouse model of in situ thromboembolic stroke and reperfusion. Stroke 2007; 38: 2771-2778.

14. Orset C, Haelewyn B, Allan SM, Ansar S, Campos F, Cho TH et al. Efficacy of alteplase in a mouse model of acute ischemic stroke: a retrospective pooled analysis. Stroke 2016; 47: 1312-1318.

15. Badaut J, Fukuda AM, Jullienne A, Petry KG. Aquaporin and brain diseases. Biochim Biophys Acta 2014; 1840: 1554-1565.

16. Mackenzie F, Ruhrberg C. Diverse roles for VEGF-A in the nervous system. Development 2012; 139: 1371-1380.

17. Sun Y, Jin K, Xie L, Childs J, Mao XO, Logvinova A et al. VEGF-induced neuroprotection, neurogenesis, and angiogenesis after focal cerebral ischemia. J Clin Invest 2003; 111 1843-1851.

18. Yurchenco PD, Patton BL. Developmental and pathogenic mechanisms of basement membrane assembly. Curr Pharm Des 2009; 15: 1277-1294.

19. Myers JC, Dion AS, Abraham V, Amenta PS. Type XV collagen exhibits a widespread distribution in human tissues but a distinct localization in basement membrane zones. Cell Tissue Res 1996; 286: 493-505.

20. Terao S, Yilmaz G, Stokes KY, Ishikawa M, Kawase T, Granger DN. Inflammatory and injury responses to ischemic stroke in obese mice. Stroke 2008; 39: 943-950.

21. Offner $H$, Subramanian S, Parker SM, Afentoulis ME, Vandenbrak AA, Hurn PD. Experimental stroke induces massive, rapid activation of the peripheral immune system. $J$ Cereb Blood Flow Metab 2006; 26: 654-665.

22. Montaner J, Salat D, García-Berrocoso T, Molina CA, Chacón P, Ribó M et al. Reperfusion therapy for acute stroke improves outcome by decreasing neuroinflammation. Transl Stroke Res 2010; 1: 261-267.
23. Shaafi S, Sharifipour E, Rhamanifar R, Hejazi S, Andalib S, Nikanfar M et al. Interleukin-6, a reliable prognostic factor for ischemic stroke. Iran J Neurol 2014; 13: 70-76.

24. Kumai $\mathrm{Y}$, Ooboshi H, Takada J, Kamouchi M, Kitazono T, Egashira K et al. Anti-monocyte chemoattractant protein-1 gene therapy protects against focal brain ischemia in hypertensive rats. J Cereb Blood Flow Metab 2004; 24: 1359-1368.

25. Hughes PM, Allegrini PR, Rudin M, Perry VH, Mir AK, Wiessner C. Monocyte chemoattractant protein-1 deficiency is protective in a murine stroke model. $J$ Cereb Blood Flow Metab 2002; 22: 308-317.

26. Strecker JK, Minnerup J, Gess B, Ringelstein EB, Schäbitz WR, Schilling M. Monocyte chemoattractant protein-1-deficiency impairs the expression of IL-6, IL-1 $\beta$ and G-CSF after transient focal ischemia in mice. PLoS One 2011; 6: e25863.

27. Strecker JK, Minnerup J, Schütte-Nütgen K, Gess B, Schäbitz WR, Schilling M. Monocyte chemoattractant protein-1-deficiency results in altered blood-brain barrier breakdown after experimental stroke. Stroke 2013; 44: 2536-2544.

28. Chen Y, Hallenbeck JM, Ruetzler C, Bol D, Thomas K, Berman NE et al. Overexpression of monocyte chemoattractant protein 1 in the brain exacerbates ischemic brain injury and is associated with recruitment of inflammatory cells. J Cereb Blood Flow Metab 2003; 23: 748-755.

29. Loddick SA, Turnbull AV, Rothwell NJ. Cerebral interleukin-6 is neuroprotective during permanent focal cerebral ischemia in the rat. J Cereb Blood Flow Metab 1998; 18 : 176-179.

30. Clark WM, Rinker LG, Lessov NS, Hazel K, Hill JK, Stenzel-Poore M et al. Lack of interleukin6 expression is not protective against focal central nervous system ischemia. Stroke 2000; 31: $1715-1720$.

31. Lemarchant $\mathrm{S}$, Pruvost M, Hébert $\mathrm{M}$, Gauberti M, Hommet $\mathrm{Y}$, Briens $\mathrm{A}$ et al. tPA promotes ADAMTS-4-induced CSPG degradation, thereby enhancing neuroplasticity following spinal cord injury. Neurobiol Dis 2014; 66: 28-42.

32. Bukhari N, Torres L, Robinson JK, Tsirka SE. Axonal regrowth after spinal cord injury via chondroitinase and the tissue plasminogen activator (tPA)/plasmin system. J Neurosci 2011; 31: 14931-14943.

33. Ortega N, Werb Z. New functional roles for non-collagenous domains of basement membrane collagens. J Cell Sci 2002; 115: 4201-4214.

34. Marneros AG, Olsen BR. The role of collagen-derived proteolytic fragments in angiogenesis. Matrix Biol 2001; 20: 337-345.

35. Hill JJ, Jin K, Mao XO, Xie L, Greenberg DA. Intracerebral chondroitinase ABC and heparan sulfate proteoglycan glypican improve outcome from chronic stroke in rats. Proc Natl Acad Sci USA 2012; 109: 9155-9160.

36. Soleman S, Yip PK, Duricki DA, Moon LD. Delayed treatment with chondroitinase ABC promotes sensorimotor recovery and plasticity after stroke in aged rats. Brain 2012; 135: $1210-1223$.

37. Lee B, Clarke D, Al Ahmad A, Kahle M, Parham C, Auckland L et al. Perlecan domain V is neuroprotective and proangiogenic following ischemic stroke in rodents. J Clin Invest 2011; 121: 3005-3023.

38. Bix GJ, Gowing EK, Clarkson AN. Perlecan domain V is neuroprotective and affords functional improvement in a photothrombotic stroke model in young and aged mice. Trans/ Stroke Res 2013; 4: 515-523.

39. Greenberg DA, Jin K. Vascular endothelial growth factors (VEGFs) and stroke. Cell Mol Life Sci 2013; 70: 1753-1761.

40. Lemarchant S, Dunghana H, Pomeshchik Y, Leinonen H, Kolosowska N, Korhonen P et al Anti-inflammatory effects of ADAMTS-4 in a mouse model of ischemic stroke. Glia 2016; 64: 1492-1507.

41. Kim DJ, Murray IA, Burns AM, Gonzales FJ, Perdew GH, Peters JM. Peroxisome proliferatoractivated receptor-beta/delta inhibits epidermal cell proliferation by down-regulation of kinase activity. J Biol Chem 2005; 280: 9519-9527.

42. Duka T, Anderson SM, Collins Z, Raghanti MA, Ely JJ, Hof PR et al. Synaptosomal lactate dehydrogenase isoenzyme composition is shifted toward aerobic forms in primate brain evolution. Brain Behav Evol 2014; 83: 216-230.

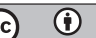

Cell Death and Disease is an open-access journal published by Nature Publishing Group. This work is licensed under a Creative Commons Attribution 4.0 International License. The images or other third party material in this article are included in the article's Creative Commons license, unless indicated otherwise in the credit line; if the material is not included under the Creative Commons license, users will need to obtain permission from the license holder to reproduce the material. To view a copy of this license, visit http://creativecommons.org/licenses/by/4.0/

(C) The Author(s) 2017 